\title{
La expansión del Pensamiento Computacional en Uruguay
}

\section{The expansion of Computational Thinking in Uruguay.}

\author{
José Miguel García. \\ Facultad Latinoamericana de Ciencias Sociales. FLACSO. Montevideo, Uruguay. \\ jmgarcia@flacso.edu.uy
}

\begin{abstract}
Resumen
En los últimos años se han desarrollado en Uruguay diversas acciones tendientes a expandir el Pensamiento Computacional en la educación primaria y media. Este movimiento no es nuevo, y está fundamentado por los trabajos que Seymour Papert llevó adelante durante décadas, apoyados fuertemente en el lenguaje Logo en sus distintas versiones.

El presente artículo realiza un recorrido por las acciones más significativas llevadas a cabo en el país desde la década de los 80, profundizando en las que actualmente se llevan a cabo con el objeto de fomentar el desarrollo del Pensamiento Computacional. En particular se presentan datos preliminares de una encuesta que se desarrolló para docentes e instituciones tendientes a relevar las actividades que se realizan en nuestro país.

En síntesis, se parte de la conceptualización de Papert(1980) y de las dificultades presentadas para el desarrollo computacional en esa época, se describe la evolución del Pensamiento Computacional como conceptualización y las acciones más relevantes realizadas en Uruguay hasta la actualidad, procurando evidenciar que en en este país se están alcanzado las proyecciones que Paper presentó originalmente.
\end{abstract}

Palabras clave: Pensamiento Computacional, Uruguay, Educación.

\begin{abstract}
In recent years, various actions have been developed in Uruguay to expand Computational Thinking in primary and secondary education. This movement is not new, and is based on the work that Seymour Papert carried out for decades, strongly supported by the Logo language in its different versions.

This article makes a tour of the most significant actions developed in the country from 1980 to the present, delving into those that are currently carried out in order to promote the development of Computational Thinking. In particular, preliminary data from a survey of teachers and institutions to find out the activities carried out in our country is presented.

In summary, it's based on the Paper's conceptualization (1980) and the difficulties presented for computational development at that time, the evolution of Computational Thinking and the most relevant actions carried out in Uruguay to date are described, trying to show that in this country the projections that Paper originally presented are being achieved.
\end{abstract}

Keywords: Computational Thinking, Uruguay, Education.

RED. Revista de Educación a Distancia. Núm. 63, Vol. 20. Artíc. 6, 30-04-2020

DOI: http://dx.doi.org/10.6018/red.410441 
RED. Revista de Educación a Distancia. Núm. 63, Vol. 20. Artíc. 6, 30-04-2020

DOI: http://dx.doi.org/10.6018/red.410441

\section{Introducción}

Si bien en la última década se ha hablado mucho sobre el Pensamiento Computacional a partir de la primera definición explícita que realiza Wing en 2006, (cuenta con más de 5.700 citas registradas en Google Académico), la expresión es mucho más antigua, encontrándose la primera referencia en Papert (1980). En dicha publicación Papert daba cuenta de las limitaciones técnicas que se encontraban en ese momento en la utilización de Logo para el desarrollo de la educación:

"En la mayoría de los casos, aunque los experimentos han sido interesantes y emocionantes, no han podido hacerlo porque eran demasiado primitivos. Sus computadoras simplemente no tenían la potencia necesaria para las actividades más atractivas y compartibles. Sus visiones de cómo integrar el pensamiento computacional en la vida cotidiana no estaban suficientemente desarrolladas. Pero habrá más intentos, y más y más. Y eventualmente, en algún lugar, todas las piezas se unirán y se "atraparán". Uno puede estar seguro de esto porque tales intentos no serán experimentos aislados operados por investigadores que pueden quedarse sin fondos o simplemente desilusionarse y renunciar. Serán manifestaciones de un movimiento social de personas interesadas en la computación personal, interesadas en sus propios hijos e interesadas en la educación.” (Papert: 1980. Pág. 182)

En dicha publicación Papert menciona el concepto de Pensamiento Computacional, aunque sin definirlo, asociándolo con otros conceptos que utilizaba frecuentemente como Pensamiento Matemático, o Pensamiento Geométrico. En sus escritos se refiriere a la capacidad de los niños de pensar en "cómo piensa" una computadora, y proponiendo la visión alternativa del niño programando un ordenador, teoría en boga en esa época, a partir de la máquina de enseñar de Skinner, del computador programando al niño (Urbina, 1999). Un interesante análisis de la robótica educativa y el pensamiento computacional a partir de los conceptos de Papert puede encontrarse en el artículo Educational Robots and Computational Thinking, de Dave Catlin y John Woollard (2014)

La primera definición de Pensamiento Computacional que plantea Wing (2006) refiere a que:

El Pensamiento Computacional implica resolver problemas, diseñar sistemas y comprender el comportamiento humano, basándose en los conceptos fundamentales de la ciencia de la Computación. El Pensamiento Computacional incluye una amplia variedad de herramientas mentales que reflejan la amplitud del campo de la Computación. Wing (2006)

En el mismo artículo plantea asimismo que "El pensamiento computacional es una habilidad fundamental para todos, no solo para los informáticos. A la lectura, la escritura y la aritmética, debemos agregar el pensamiento computacional a la capacidad analítica de cada niño." Wing (2006), sentando las bases de que este tipo de enfoques va más allá de la intención de generar futuros programadores, tal como se sostiene en un artículo previo de esta misma revista: "Entendemos que la robótica educativa entonces 
RED. Revista de Educación a Distancia. Núm. 63, Vol. 20. Artíc. 6, 30-04-2020

DOI: http://dx.doi.org/10.6018/red.410441

no debe ser vista como privativa para los alumnos que trabajarán en áreas técnicas, sino como dispositivo pedagógico para un aprendizaje creativo.” (García 2015).

A partir de dicha definición se realizan múltiples interpretaciones, entre ellas de la propia Wing (2008) o Wing (2011), que procuran ajustarla a un concepto que se encuentra aún en construcción.

Una definición más elaborada y actualizada de Pensamiento Computacional la proporciona Zapata Ros:

El pensamiento computacional consiste en la resolución de problemas, el diseño de los sistemas, y la comprensión de la conducta y de las actividades humanas haciendo uso de conceptos y procedimientos básicos para el trabajo y la elaboración de programas y algoritmos en la informática, valiéndose para ello de habilidades específicas y de técnicas necesarias para estos objetivos, que en conjunto constituyen la base de la cultura digital. (Zapata Ros, 2018)

En el presente artículo esta es la definición que usamos cuando nos referimos a Pensamiento Computacional.

\section{El pensamiento computacional antes de la definición de "pensamiento computacional".}

En Uruguay el trabajo en la línea del Pensamiento Computacional, a través de la programación y la robótica, comenzó a fines de la década de los '80. Si bien la programación inició con el enfoque construccionista de Papert, luego derivó a la programación per se, perdiéndose en muchos casos el desarrollo del Pensamiento Computacional, y enfocándose en la programación primero, y en el uso de la ofimática luego, tal como lo hemos descrito en el artículo Educación y tecnología. Construyendo preguntas. (García y Castrillejo, 2006). Sin embargo, una línea del trabajo de Papert continuó avanzando, apoyada fundamentalmente por la Asociación Logo del Uruguay, lo que llevó a los primeros trabajos con robótica educativa a principios de los años 90. A modo de ejemplo, en 1994 y 1997 se desarrollaron proyectos conjuntos con escuelas de Suiza, Dinamarca, Estados Unidos de América y Uruguay en el marco del proyecto PanGea ${ }^{1}$, donde entre distintos centros educativos se construyeron colaborativamente soluciones a problemas ecológicos, se maquetaron en dispositivos robóticos y fueron comandadas desde los otros países integrantes del proyecto. Participó en esta instancia el propio Seymour Papert, desde Boston.

A partir de 1999 se desarrolla anualmente la RoboTeca, un encuentro de instituciones educativas que trabajan con programación y robótica, como forma de incentivar este tipo de trabajo, así como fomentar los intercambios tanto entre estudiantes como entre docentes, en un formato de aprendizaje compartido. Todas estas acciones se llevaron adelante en sus comienzos por centros educativos privados, y unos pocos centros públicos que contaban con el equipamiento necesario y con docentes fuertemente involucrados en el desarrollo educativo a través del uso pedagógico de la tecnología.

1 Página del proyecto: https://tecfa.unige.ch/proj/pangea/historique/pangee2.html

La expansión del Pensamiento Computacional en Uruguay. José Miguel García.

Pág. 3 de 15 
En esta etapa la cantidad de centros educativos involucrados no superaban los 50 en todo el país, estableciéndose una meseta de 15 años en cuanto al número de participantes (García, 2013). En la actualidad no existen estudios que permitan caracterizar en forma cuantitativa esta etapa, que estuvo fuertemente marcada por iniciativas docentes y de unos pocos centros educativos, sin políticas públicas que las sustentaran.

En síntesis, los trabajos realizados en Uruguay a partir de los años 80 y antes que la definición de Wing fuera propuesta el 2006, presentan múltiples iniciativas con el enfoque de Seymour Papert en Pensamiento Computacional en pequeña escala.

\section{En la era del Pensamiento Computacional}

El Plan Ceibal ${ }^{2}$ comienza a funcionar en Uruguay en el año 2007, a partir de la entrega de los primeros laptops a estudiantes y docentes de la escuela Italia de Villa Cardal. En 2009 se alcanza la universalización a la escuela primaria pública de todo el país, y a partir de 2010 se comienza la implementación en la enseñanza media.

Esto se vio potenciado por el despliegue de cobertura de Internet, que es proporcionada por la Administración Nacional de Telecomunicaciones (ANTEL), que permite que cada centro educativo tenga conectividad, alcanzando además el $100 \%$ de conexiones a través de fibra óptica en todos los centros urbanos del país.

Los primeros dispositivos entregados fueron las XO de $\mathrm{OLPC}^{3}$ que incluyeron en su configuración los programas TortugArte, e-toys y luego Scratch. Esto potenció el trabajo con herramientas de programación, puesto que los docentes re-descubrieron sus cualidades educativas. El proceso de universalización en la enseñanza pública fue en un corto plazo ( 3 años), las preocupaciones de las autoridades estuvieron más abocadas al funcionamiento general que a las iniciativas individuales, por lo que no existen registros que permitan cuantificar los usos orientados hacia el pensamiento computacional.

En 2009 inicia el Proyecto Butiá ${ }^{4}$, desarrollado desde la Facultad de Ingeniería de la Universidad de la República, que tiene como objetivo la creación de dispositivos de hardware y software abierto para el desarrollo de la robótica educativa a nivel de enseñanza primaria y media.

El proyecto de robótica educativa en forma masiva surge en 2010, año en el que se desarrolla un piloto en 10 centros de la educación pública del Uruguay, y se expandió en los años siguientes. A partir de ese momento se implementan los llamados Laboratorios de Tecnologías Digitales (LabTeD), con el fin de propiciar los cambios de los espacios dedicados a la enseñanza de la informática para transformarlos en espacios de trabajo en proyectos que involucren tecnologías digitales, donde la programación y la robótica tienen un papel preponderante.

2 Proyecto de Un Laptop por Alumno en Uruguay. https://www.ceibal.edu.uy/es Puede accederse a diversas publicaciones académicas en la página de la Administración Nacional de Educación Pública, http://anep.edu.uy/publicaciones?page=1

3 http://one.laptop.org/

4 https://www.fing.edu.uy/inco/proyectos/butia/ 
RED. Revista de Educación a Distancia. Núm. 63, Vol. 20. Artíc. 6, 30-04-2020

DOI: http://dx.doi.org/10.6018/red.410441

\section{Semana de la Robótica y la Programación en la Educación ${ }^{5}$}

A partir del año 2013 se comienzan a desarrollar actividades de intercambio y difusión masivas, que involucraron a proyectos de robótica y programación, desde la Administración Nacional de Enseñanza Pública (ANEP)

Ese año se realizó el primer Día Mundial del TortugArte, que tuvo su edición en Uruguay, con una asistencia pequeña y centralizada en un evento en el que participaron 70 personas, entre estudiantes y docentes, y sentando las bases para futuros eventos que se desarrollaron en los años posteriores. Este formato se trasformó en 2016, con la incorporación en la organización de los Centros $\mathrm{MEC}^{6}$, en la Semana de la Robótica y la Programación en Educación, que se realiza anualmente en el Uruguay. La clave de esta actividad se encuentra en la descentralización, generando eventos en distintas localidades de todo el país.

En el primer año con este nuevo formato se realizaron 6 eventos en distintos puntos del país, y la actividad fue evolucionando hasta alcanzar los 14 eventos en 2018, y 12 en 2019 coordinados desde la ANEP, con una amplia dispersión geográfica, tal como se muestra en las figuras 1 y 2. En las últimas ediciones se superaron los 1.800 participantes en todo el país, entre docentes, estudiantes y público en general.

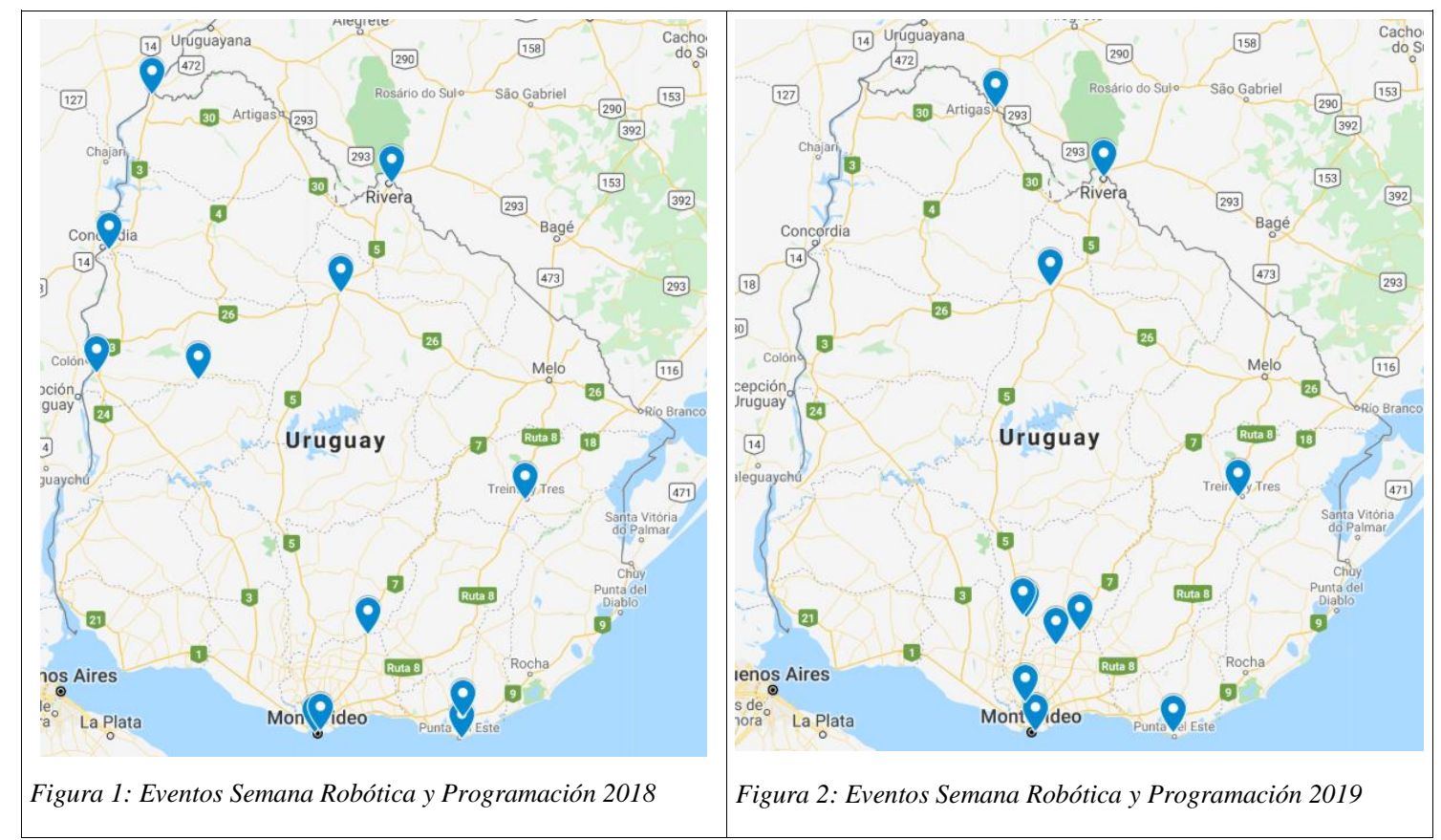

5 https://www.anep.edu.uy/destacada-1/especialista-destac-oportunidades-aprendizaje-significativopensamiento-computacional https://www.anep.edu.uy/15-d/nueva-edici-n-semana-rob-tica-y-programaci-n-en-educaci-n https://www.anep.edu.uy/15-d/semana-rob-tica-y-programaci-n-convoc-j-venes-y-docentes-en-florida https://www.anep.edu.uy/15-d/rob-tica-y-programaci-n-se-instalaron-en-tacuaremb

6 https://www.centrosmec.gub.uy/innovaportal/v/19627/31/mec/que-es-centros-mec 
RED. Revista de Educación a Distancia. Núm. 63, Vol. 20. Artíc. 6, 30-04-2020

DOI: http://dx.doi.org/10.6018/red.410441

Estos eventos son organizados a nivel local, y coordinados centralizadamente para el apoyo y la difusión. De esta manera se empodera a los actores de las distintas localidades y centros educativos de todo el país, que son quienes los gestionan según sus posibilidades e intereses. En dichos eventos, además de potenciar a los docentes locales, se estimula la realización de talleres dados por estudiantes, para estudiantes y docentes, lo que favorece las reformulaciones de los roles docente y alumno.

Para fortalecer la concepción de Pensamiento Computacional y potenciar las fortalezas locales con actividades externas, se contó en 2018 con la presencia de Miguel Zapata $\operatorname{Ros}^{7}$, de la Universidad de Murcia, quien fue el responsable del dictado de las conferencias "El Pensamiento Computacional, análisis de una competencia clave en la nueva alfabetización digital", realizadas en Montevideo, Guichón y Rivera.

En 2019 se contó con la presencia de Graciela Caldeiro ${ }^{8}$, de FLACSO Argentina, quien dictó las conferencias de "El pensamiento computacional: una oportunidad para el aprendizaje", "Tapados de tanta información: ¿qué es el Big Data?" y talleres de "Enseñar a programar con proyectos de videojuegos", en Montevideo, Florida, San Ramón, Las Piedras, Maldonado, Fray Marcos, Rivera, Tacuarembó y Treinta y Tres.

\section{Olimpíadas de Robótica, Programación y Videojuegos 9}

Desde el año 2014 se organizan, a través del Plan Ceibal, las olimpíadas de robótica, programación y videojuegos. Las mismas cuentan con una amplia difusión en todo el país, donde se realiza una pre-selección de los equipos participantes en diversas categorías. Confluyen a la actividad central que se llevan a cabo durante dos días en la ciudad de Montevideo, y en la que participan más de 1.800 estudiantes y 450 docentes de todo el país. Este dato refiere a los que finalmente participaron en la olimpíada en forma presencial, pero el número de trabajos en las distintas localidades es significativamente mayor.

Estas olimpíadas son también una instancia de presentación de trabajos de diversa índole, donde prevalece el enfoque del pensamiento computacional a través del desarrollo de proyectos por parte de los estudiantes, en torno a una temática específica cada año: "Los proyectos implican la identificación de un problema, el proceso de investigación y la elaboración de una solución apropiada para ese problema. Se valora especialmente el abordaje desde el marco conceptual de Pensamiento computacional." (Ceibal, 2019) Asimismo, dichas instancias operan también como clasificatorias a eventos internacionales de la FIRST LEGO League que se desarrollan anualmente, y en la que participan los estudiantes premiados en las respectivas categorías.

\section{Programa de Pensamiento Computacional}

En 2017 comienza un piloto de Pensamiento Computacional ${ }^{10}$ que se desarrolla desde el Plan Ceibal. Utiliza una modalidad novedosa, mediante la red de videoconferencias (VC)

7 https://www.linkedin.com/in/miguelzapata/

8 https://www.linkedin.com/in/gracielapaulacaldeiro/

9 https://www.ceibal.edu.uy/es/articulo/olimpiada-de-robotica-programacion-y-videojuegos-2019

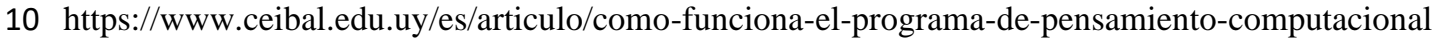


disponibles en todos los centros urbanos del país, y que ha sido ampliamente utilizada por el proyecto Ceibal en Inglés ${ }^{11}$. El mismo consiste en el desarrollo de una clase semanal con un profesor remoto a través de VC donde participan los alumnos con su docente de aula. Durante el resto de la semana se desarrollan con el docente de clase, que no necesariamente es un especialista en la materia, distintas actividades que quedan propuestas.

Este sistema permite alcanzar un gran número de centros educativos que por su dispersión geográfica no podían acceder anteriormente a estos espacios. En el caso de Ceibal en Inglés, se ha cubierto de esta forma la totalidad de los centros educativos urbanos del país. La actividad de Pensamiento Computacional propuesta inició con 100 grupos en 2017, alcanzando en 2019 los 1800 grupos, que se espera se amplíen fuertemente en 2020.

\section{Pensamiento Computacional: un proyecto interinstitucional}

Debido a la multiplicidad de actores involucrados en temáticas de pensamiento computacional, muchas veces aisladas entre sí, se estableció en 2018, a instancias de la ANEP, la Mesa Interinstitucional de Pensamiento Computacional, integrada por distintos actores de la educación y la sociedad civil. Esta mesa está liderada por el Departamento de Tecnologías Educativas de la Dirección Sectorial de Información para la Gestión y la Comunicación del Consejo Directivo Central de la Administración Nacional de Educación Pública (ANEP), representantes de cada uno de los Consejos Desconcentrados de la ANEP (Consejo de Educación Inicial y Primaria, Consejo de Educación Secundaria, Consejo de Educación Técnico Profesional y Consejo de Formación en Educación), la dirección de Educación y Centros MEC del Ministerio de Educación y Cultura, el Centro Ceibal, la Facultad de Ingeniería y el Centro Interdisciplinario en Cognición para la Enseñanza y el Aprendizaje (CICEA) de la Universidad de la República, la organización Girls in Tech Uruguay, la Administración Nacional de Telecomunicaciones (ANTEL) y la Fundación Telefónica-Movistar.

El objetivo de esta mesa de trabajo es la articulación de actividades con un fuerte anclaje territorial donde, sin perder las características de cada colectivo, se promueva el pensamiento computacional.

Una importante actividad se realizó en año 2018, cuando se recibió la visita de David Cuartielles, co-fundador de Arduino, quien participó de la mesa de trabajo interinstitucional $^{12}$ compartiendo sus experiencias y diversas propuestas educativas. Esto significó un importante hito en la difusión de Arduino en la educación uruguaya.

\section{Encuesta de Pensamiento Computacional en Educación en Uruguay}

Durante el año 2019 Mesa Interinstitucional de Pensamiento Computacional, acordó y coordinó la realización de la Primera Encuesta nacional de relevamiento de acciones

11 https://ingles.ceibal.edu.uy/

12 https://www.anep.edu.uy/15-d/grupo-t-cnico-anep-trabaja-en-aplicaci-n-pr-ctica-pensamientocomputacional-en-el-aula 
relativas a Pensamiento Computacional en Uruguay, con el fin de relevar las actividades que en esta línea se están llevando adelante en el país, puesto que no existen investigaciones que sistematicen los trabajos realizados en esta área. La encuesta está dividida en dos formularios autoadministrados de participación voluntaria: uno enfocado hacia los docentes y el otro hacia las instituciones que desarrollan este tipo de trabajo.

La encuesta para docentes indaga acerca de las fuentes de información que utiliza, los espacios de trabajo, y solicita datos del proyecto más relevante, acerca de su duración, nivel educativo, localidad, público con el que trabaja, objetivos, y si realiza evaluación de dichos espacios.

La encuesta para instituciones releva los lugares y niveles educativos donde se desarrolla la actividad, edades de los destinatarios, herramientas utilizadas y duración de la experiencia.

Si bien los resultados no fueron alentadores en cuanto a la cantidad de respuestas (601 docentes y 155 instituciones) aportó una serie de datos interesantes que están aún en proceso de análisis por la citada Mesa de Trabajo. Cabe destacar que estos datos no pueden considerarse representativos.

\section{Encuesta a docentes}

En la encuesta a docentes se solicitaba que respondieran sobre el proyecto que consideraban más significativo. Entre los datos obtenidos, se destaca que el $45 \%$ de los proyectos se desarrollan en forma anual, y el $90 \%$ se realizan en espacios de educación formal. El $65 \%$ son proyectos de programación, el $10 \%$ de robótica y el $25 \%$ de otras actividades.

Un dato interesante lo aporta la formación que señalan los entrevistados respecto al tema, según el formato de la misma. En la figura 3 se representan las respuestas acerca de las principales fuentes de formación en Pensamiento Computacional de los docentes encuestados. 
RED. Revista de Educación a Distancia. Núm. 63, Vol. 20. Artíc. 6, 30-04-2020

DOI: http://dx.doi.org/10.6018/red.410441

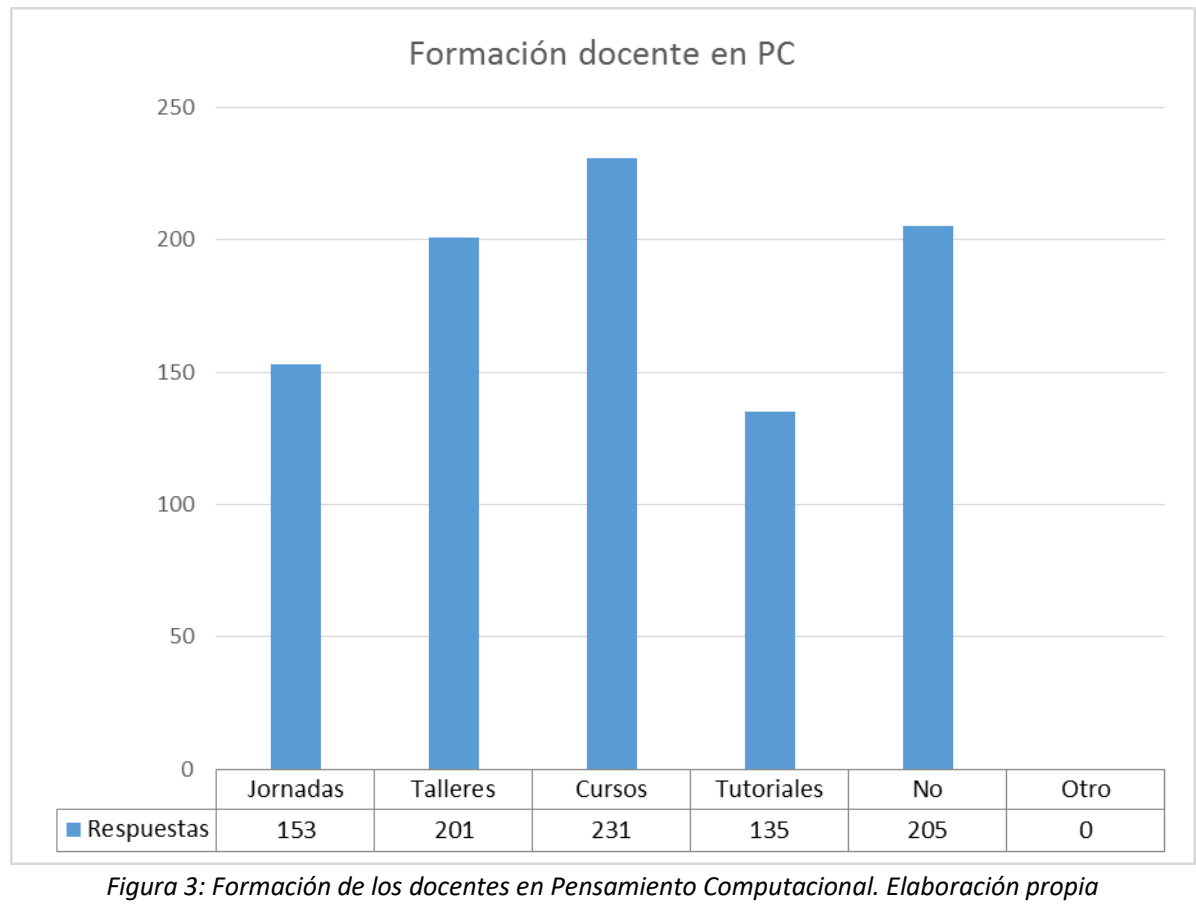

Resulta significativo que haya escasas diferencias entre quienes realizaron cursos y/o talleres con aquellos que no recibieron formación específica sobre el tema.

Desde la perspectiva docente, puede resultar llamativo que la tercera parte de los docentes encuestados señala no tener capacitación previa en Pensamiento Computacional. Esto puede explicarse por dos motivos: El programa de Pensamiento Computacional proporcionado por CEIBAL provee un profesor remoto, que es quien lidera la enseñanza, que es acompañado del docente de aula que no necesariamente domina esta temática. Por otro lado, en la línea de programación y robótica existe en Uruguay una cultura de aprender junto con los alumnos, tal como quedó evidenciado el trabajo que estudió esta situación en Uruguay (García: 2018).

Asimismo, se consultó por la evaluación de las actividades: si se realizaban, y de qué tipo era, obteniéndose los resultados que se presentan en la figura 4: 
RED. Revista de Educación a Distancia. Núm. 63, Vol. 20. Artíc. 6, 30-04-2020

DOI: http://dx.doi.org/10.6018/red.410441

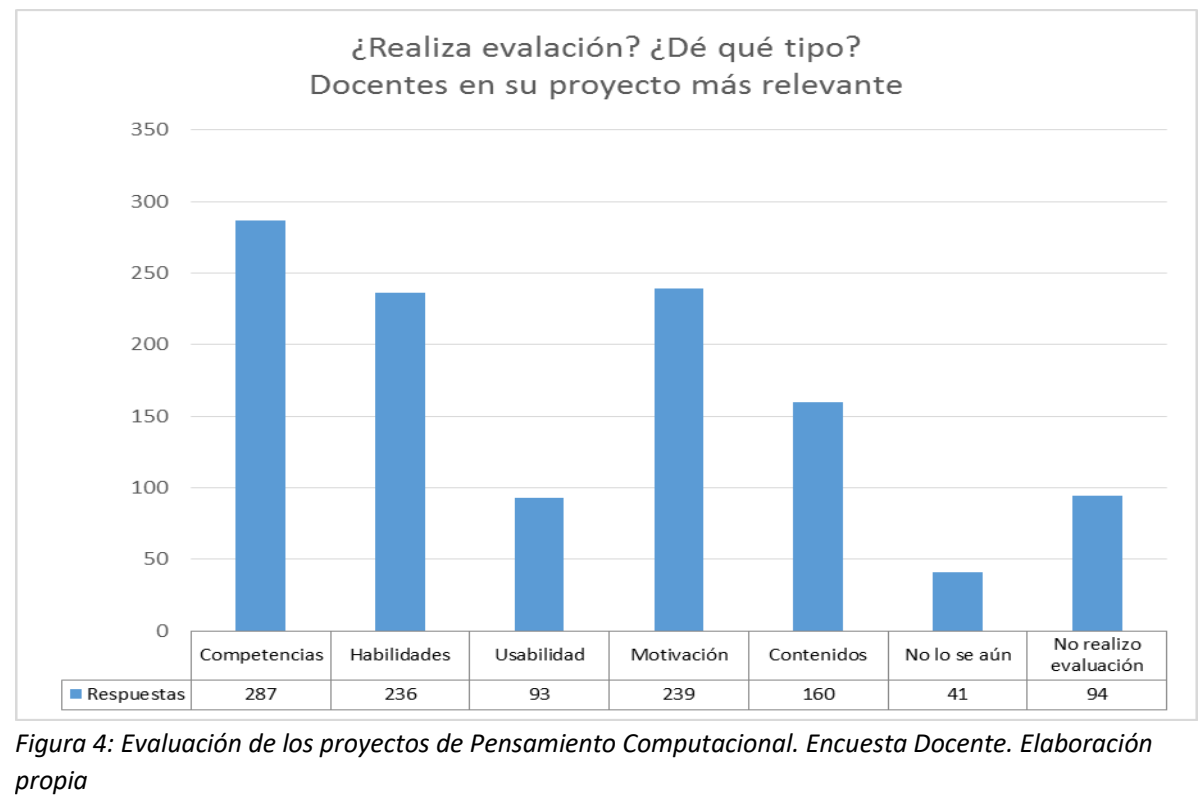

Se observa que estas actividades son más desarrolladas con enfoque de desarrollo de habilidades y competencias, con un fuerte componente motivacional, más allá de los contenidos curriculares o su funcionalidad para trabajos futuros.

\section{Encuesta a instituciones}

Debido a la escasa respuesta de las instituciones (existen 2.841 centros educativos públicos), desde la ANEP se tomó la iniciativa de realizar nuevamente el relevamiento institucional a través de encuestas telefónicas con los centros educativos, alcanzándose en esta ocasión la mayoría de los centros educativos públicos, exceptuando las escuelas primarias rurales, lo que lo configura más como un censo que como una encuesta. Teniendo en cuenta que las escuelas rurales (1.085) representan el 38\% de las escuelas públicas, pero atienden solamente al 5,7\% de la población escolar, además de contar con dinámicas propias, se optó por excluirlas de este primer relevamiento debido a que el tiempo no permitía realizar la encuesta a todos los centros educativos públicos antes de la finalización de los cursos.

La encuesta telefónica realizada a las instituciones aporta también datos relevantes. El primero de ellos es que en el $44 \%$ de los centros encuestados se desarrollan trabajos relativos a Pensamiento Computacional. Si se considera el carácter voluntario de estos trabajos y el poco tiempo transcurrido desde que comenzó el proceso de masificación en esta línea de trabajo, resulta un número que se encuentra por encima de lo esperado. Si se desagregan los datos, este valor está en el entorno del 50\% para los centros educativos públicos de enseñanza media y en un 33\% para los centros de formación docente.

Asimismo, el $72 \%$ de estos centros trabajó con programación, el 52\% con robótica y el $9 \%$ en otras modalidades (respuestas no excluyentes).

La expansión del Pensamiento Computacional en Uruguay. José Miguel García. Pág. 10 de 15 
Siendo esta encuesta una primera aproximación al relevamiento, con el objetivo de conocer quiénes son y dónde están los docentes que trabajan pensamiento computacional, se planea para las siguientes ediciones valorar otros aspectos vinculados a su desarrollo específico, como en qué espacios escolares los llevan a cabo, si se realiza vinculado con temas curriculares específicos, etc.

\section{A modo de cierre}

En Uruguay el Plan Ceibal provee de tabletas o computadoras para todos los estudiantes y docentes de la educación primaria y media básica pública, además de conectividad a internet en los centros educativos, y planes de conectividad gratuita tanto en lugares públicos como en hogares de bajos recursos. Este despliegue permite alcanzar la expansión del trabajo en pensamiento computacional, que ya supera el $45 \%$ de los centros educativos públicos.

Si se observa el contexto regional, el Pensamiento Computacional comienza a ser una preocupación de los gobiernos, siendo obligatoria en Argentina, desde 2018, la programación y la robótica en la educación básica (Vázquez et al: 2019).

Uruguay no tiene la obligatoriedad del trabajo en Pensamiento Computacional en la educación. La participación es voluntaria, y variable dependiendo de los docentes, los centros educativos y los dispositivos disponibles. Al ser de carácter voluntario y en muchos casos desarrollado por los docentes dentro del aula como parte de sus tareas curriculares es difícil tener una dimensión clara de su avance. Sin embargo, se entiende que con el compromiso de docentes e instituciones llega a niveles significativos (casi la mitad de los centros educativos públicos) en escaso tiempo (10 años de la universalización de las tecnologías en la educación pública). En este sentido se puede afirmar que se alcanza la dimensión de movimiento social que sugería Papert en 1980, citado al comienzo del presente artículo:

"Pero habrá más intentos, y más y más. Y eventualmente, en algún lugar, todas las piezas se unirán y se "atraparán". Uno puede estar seguro de esto porque tales intentos no serán experimentos aislados operados por investigadores que pueden quedarse sin fondos o simplemente desilusionarse y renunciar.Serán manifestaciones de un movimiento social de personas interesadas en la computación personal, interesadas en sus propios hijos e interesadas en la educación.” (Papert: 1980. Pág. 182)

La expansión que ha tenido el Pensamiento Computacional en Uruguay no significa que todo la tarea esté concluida, pero en el transcurso de poco más de una década desde el inicio de trabajos con programación, robótica y/o pensamiento computacional realizados en forma masiva, representa un alcance significativo, a la vez que desafiante para las acciones futuras tendientes a alcanzar la universalización de esta línea de trabajo. 
Como forma de sistematizar y compartir experiencias educativas, se realiza el concurso para la publicación "Sembrando Experiencias"13 que realiza anualmente el Departamento de Tecnología Educativa del Consejo Directivo Central de la Administración Nacional de Educación pública. El último llamado se realizó en el marco del Pensamiento Computacional. ${ }^{14}$

Este hecho, sumado a la nueva realización de la encuesta a docentes e instituciones, que será ampliada y revisada en 2020, permitirá obtener datos más actualizados del avance del Pensamiento Computacional en la educación uruguaya.

Presentación del manuscrito: 15 de enero de 2020

Fecha de aprobación: 23 de marzo de 2020

Fecha de publicación: 30 de abril de 2020

García, J. M. (2020). La expansión del Pensamiento Computacional en Uruguay. RED. Revista Educación a Distancia, 20(63). http://dx.doi.org/10.6018/red.410441

\section{Financiación}

Este trabajo no ha recibido ninguna subvención específica de los organismos de financiación en los sectores públicos, comerciales o sin fines de lucro.

\section{Referencias}

Bender, W. (2017). The Sugar Learning Platform: Affordances for Computational Thinking. RED. Revista de Educación a Distancia, 54. Consultado el (10/03/2020) en http://www.um.es/ead/red/

Catlin, D. and Woollard, J. (2014), Educational Robots and Computational Thinking, Proceedings of 4th International Workshop Teaching Robotics, Teaching with Robotics \& 5th International Conference Robotics in Education Padova (Italy) July 18, 2014, pp. 144-151, disponible en https://www.researchgate.net/profile/Mohamed_Mourad_Lafifi/post/robots_and_c omputational_thinking/attachment/5ba68fe83843b006753aa23d/AS\%3A6737355 $14267649 \% 401537642472489 /$ download/Educational+Robots+and+Computation al+Thinking.pdf

Ceibal (2019) Olimpíada de Robótica, Programación y Videojuegos 2019. Disponible en https://www.ceibal.edu.uy/es/articulo/olimpiada-de-robotica-programacion-yvideojuegos-2019

García, J. M. y Castrillejo, D. (2006) Educación y tecnología. Construyendo preguntas. En Martinis, Pablo (comp): Pensar la escuela más allá del Contexto. Psicolibros

$13 \mathrm{http}: / / \mathrm{www} \cdot$ anep.edu.uy/sembrando/

14 https://uruguayeduca.anep.edu.uy/noticias/3594

La expansión del Pensamiento Computacional en Uruguay. José Miguel García. Pág. 12 de 15 
Waslala, Montevideo. en http://www.argos.edu.uy/sitio/documentos/educacion_y_tecnologia.pdf

García, J. M. (2013) Hacia la masificación de la robótica educativa. En Anales del II Encuentro Internacional del Conocimiento: diálogos en nuestra América / II Encuentro de las Ciencias Humanas y Tecnológicas para la Integración en el Conosur, Bogotá, 2013. Disponible en http://www.argos.edu.uy/sitio/documentos/Garcia_Jose_Miguel_Hacia_la_masifi cacion_de_la_robotica_educativa.pdf

García, J. M. (2015). La programación como parte de un proceso educativo. RED. Revista de Educación a Distancia, 46(8). 15 de Septiembre de 2015. Consultado el 12/12/2019 en http://www.um.es/ead/red/46

García, J. M. (2018) ¿Es posible enseñar cuando no se sabe? Estrategias y metodologías utilizadas por docentes en estas situaciones (Tesis de maestría) Universidad Nacional de Córdoba. Disponible en http://www.argos.edu.uy/sitio/documentos/Garcia_Es_posible_ense\%C3\%B1ar_c uando_no_se_sabe.pdf

Urbina Ramírez, S. (1999). Informática y teorías del aprendizaje. Pixel-Bit. Revista de Medios y Educación, 12, 87-100. Disponible en http://hdl.handle.net/11441/45480

Vázquez Uscanga, E. A., Bottamedi, J. y Brizuela, M. L. (2019) Pensamiento computacional en el aula: el desafío en los sistemas educativos de Latinoamérica. Revista Interuniversitaria de Investigación en Tecnología Educativa (RIITE) № 7. $\begin{array}{llllll}\text { Diciembre } 2019 & \text { pp. } 36-47 & \text { ISSN: } & 2529-9638 & \text { DOI: }\end{array}$ http://dx.doi.org/10.6018/riite.397901

Wing, Jeannette M (2006) Computational Thinking en Viewpoint COMMUNICATIONS OF THE ACM March 2006/Vol. 49, No. 3. Disponible en http://www.cs.cmu.edu/afs/cs/usr/wing/www/publications/Wing06.pdf

Wing, Jeannette M (2008) Computational thinking and thinking about computing, 366Philosophical Transactions of the Royal Society A: Mathematical, Physical and Engineering Sciences. Disponible en https://doi.org/10.1098/rsta.2008.0118

Wing, Jeannette M (2011) Research Notebook: Computational Thinking--What and Why? The magazine of the Carnegie Mellon University School of Computer Science, disponible en http://people.cs.vt.edu/ kafura/CS6604/Papers/CT-WhatAnd-Why.pdf

Zapata Ros, M. (2018) Pensamiento computacional. Una tercera competencia clave (III): ¿Qué es el Pensamiento Computacional? Una definición. Blog RED de Hypotheses. El aprendizaje en la Sociedad del Conocimiento. Disponible en https://red.hypotheses.org/tag/definicion-de-pensamiento-computacional 


\section{Recursos:}

Se detallan a continuación una serie de enlaces a recursos que han sido utilizados como referencias a lo largo del artículo, y que permiten ampliar la información de las actividades descriptas.

Proyecto PanGea:

https://tecfa.unige.ch/proj/pangea/historique/pangee2.html

Plan Ceibal. Proyecto de Un Laptop por Alumno en Uruguay:

https://www.ceibal.edu.uy/es

Publicaciones sobre Plan Ceibal:

Centro Ceibal: https://www.ceibal.edu.uy/es/publicaciones

Administración Nacional de Educación Pública:

http://anep.edu.uy/publicaciones?page $=1$

OLPC (One lapotop per child):

http://one.laptop.org/

Proyecto Butiá (Facultad de Ingeniería, Universidad de la República):

https://www.fing.edu.uy/inco/proyectos/butia/

Semana de la Robótica y la Programación en Educación. Administración Nacional de Educación Pública:

https://www.anep.edu.uy/destacada-1/especialista-destac-oportunidadesaprendizaje-significativo-pensamiento-computacional

https://www.anep.edu.uy/15-d/nueva-edici-n-semana-rob-tica-y-programaci-nen-educaci-n

https://www.anep.edu.uy/15-d/semana-rob-tica-y-programaci-n-convoc-j-venesy-docentes-en-florida

https://www.anep.edu.uy/15-d/rob-tica-y-programaci-n-se-instalaron-entacuaremb

Centros MEC (Ministerio de Educación y Cultura):

https://www.centrosmec.gub.uy/innovaportal/v/19627/31/mec/que-es-centrosmec

Plan Ceibal: Olimpíadas de robótica, programación y videojuegos:

https://www.ceibal.edu.uy/es/articulo/olimpiada-de-robotica-programacion-yvideojuegos-2019

Plan Ceibal: Proyecto de Pensamiento Computacional:

La expansión del Pensamiento Computacional en Uruguay. José Miguel García. Pág. 14 de 15 
RED. Revista de Educación a Distancia. Núm. 63, Vol. 20. Artíc. 6, 30-04-2020

DOI: http://dx.doi.org/10.6018/red.410441

https://www.ceibal.edu.uy/es/articulo/que-aporta-al-aula-el-pensamientocomputacional https://www.ceibal.edu.uy/es/articulo/como-funciona-el-programa-depensamiento-computacional

Plan Ceibal: Proyecto Ceibal en Inglés:

https://ingles.ceibal.edu.uy/

Mesa de Trabajo Interinstitucional sobre Pensamiento Computacional:

https://www.anep.edu.uy/15-d/grupo-t-cnico-anep-trabaja-en-aplicaci-n-pr-cticapensamiento-computacional-en-el-aula

Mesa de Trabajo Interinstitucional: Visita de David Cuartielles:

https://www.anep.edu.uy/15-d/grupo-t-cnico-anep-trabaja-en-aplicaci-n-pr-cticapensamiento-computacional-en-el-aula https://www.anep.edu.uy/15-d/aprendizaje-por-proyectos-otra-forma-acercarseal-conocimiento

Encuesta de Pensamiento Computacional:

https://www.anep.edu.uy/15-d/encuesta-sobre-pensamiento-computacional-eneducaci-n-estar-disponible-hasta-el-15-agosto

La expansión del Pensamiento Computacional en Uruguay. José Miguel García. Pág. 15 de 15 